\title{
Aortic Pseudoaneurysm Following Aortic Valve Replacement Complicated by Sternal Wound Infection
}

\author{
James Keiler ${ }^{1}$, Erik Scott ${ }^{1}$, Timothy George ${ }^{1}$, and Gorav Ailawadi ${ }^{1}$ \\ ${ }^{1}$ University of Virginia
}

March 21, 2021

\begin{abstract}
Pseudoaneurysm of the ascending aorta is a rare complication of cardiac surgery occurring in less than $0.5 \%$ of cases. Deep sternal wound infections (DSWI) increase the likelihood of aortic pseudoaneurysm. An ascending aortic pseudoaneurysm has high morbidity and the mortality rate has ranged from $29 \%-46 \%$ in the medical literature. We report a complex patient who underwent an AVR at an outside hospital complicated by DSWI requiring pectoral flap coverage. Months later, he was transferred to our institution with acute swelling of his sternum, diagnosed as massive aortic pseudoaneurysm originating from the cannulation site.
\end{abstract}

Aortic Pseudoaneurysm Following Aortic Valve Replacement Complicated by Sternal Wound Infection Authors : James Keiler BS ${ }^{1}$; Erik Scott MD MS${ }^{1}$; Timothy George MD ${ }^{1}$; Gorav Ailawadi MD MBA ${ }^{1,2}$

Institutions and Affiliations :

${ }^{1}$ Division of Cardiac Surgery, University of Virginia, Charlottesville, Virginia

${ }^{2}$ Department of Cardiac Surgery, University of Michigan, Ann Arbor, Michigan

Classification : Case Report

Running Title: Pseudoaneurysm Following Valve Replacement

Corresponding Author:

Gorav Ailawadi, MD, MBA

1500 E Medical Center Drive / CVC 5144

Ann Arbor, MI 48109

ailawadi@umich.edu

(734)-936-4974

Funding: None

Data sharing not applicable - no new data generated

\section{Abstract}

Pseudoaneurysm of the ascending aorta is a rare complication of cardiac surgery occurring in less than $0.5 \%$ of cases $^{1}$. Deep sternal wound infections (DSWI) increase the likelihood of aortic pseudoaneurysm. An ascending aortic pseudoaneurysm has high morbidity and the mortality rate has ranged from $29 \%-46 \%$

${ }^{2-4}$. We report a complex patient who underwent an aortic valve replacement (AVR) at an outside hospital 
complicated by DSWI requiring bilateral pectoralis major flap coverage. Months later, he was transferred to our institution with acute swelling of his sternum, diagnosed as massive aortic pseudoaneurysm originating from the cannulation site.

\section{Introduction}

Pseudoaneurysm of the aorta is a rare complication of cardiac surgery. Approach to repair is largely patient dependent. We present a case in which an aortic pseudoaneurysm developed following an aortic valve replacement (AVR) complicated by deep sternal wound infection (DSWI). This report was granted a waiver exemption by the Institutional Review Board at the University of Virginia due to the anonymous nature of the single-patient case report.

\section{Case Summary}

We report a 64 yo male with a history of severe aortic stenosis who underwent aortic valve replacement in 2015 with a $21 \mathrm{~mm}$ Mitroflow bioprosthetic valve (Sorin, Arvada, CO) at an outside hospital. The postoperative course was complicated by pan-sensitive Serratia Marcescens deep sternal wound infection and mediastinitis requiring multiple debridements and treatment with a six-week course of intravenous vancomycin and ceftriaxone. He subsequently required a sternal reconstruction using bilateral pectoralis major flaps and a right rectus abdominis flap. He acutely did well from this hospitalization. However, he then presented three months after his index surgery with 2-3 day history of fevers, chills, and a new sternal mass. His surgeon considered acutely decompressing the mass at the bedside but decided to transfer the patient to our institution.

Upon arrival at our institution he was neurologically intact with an EKG showing sinus tachycardia, all other vitals within normal limits. His exam was impressive with a rapidly enlarging, erythematous, anterior chest wall mass (Figure 1, 2 ). An urgent computed tomographic angiogram (CTA) scan of the chest demonstrated a blush at the aortic cannulation site with active arterial extravasation into a massive pseudoaneurysm of the distal ascending aorta filling the entire anterior mediastinum (Figure 3 ). Echocardiogram demonstrated grossly normal left ventricle (LV) systolic function with poor visualization of the ascending aorta. Percutaneous coverage of the pseudoaneurysm as a temporizing measure was considered but not feasible due to a lack of distal landing zone or a small enough neck.

Given the rapid expansion, he was taken emergently to the operating room for repair. The right femoral vein and artery were cannulated and patient was cooled. Due to several small aortic paravalular leaks and concern for LV distension, a left anterior thoracotomy was performed and an LV vent placed directly through the LV apex. Manual compression was also required to keep the LV from distending.

By the time the patient's temperature reached $28^{\circ} \mathrm{C}$ with the $\mathrm{LV}$ continuing to distend, a redo sternotomy was performed. Upon entering the anterior mediastinum, the large pseudoaneurysm was grossly entered with evidence if acute arterial hemorrhage. Circulatory arrest was initiated and the aorta was rapidly dissected out to identify the aortic cannulation site where multiple, pledgeted sutures were identified loosely attached at the original cannulation site. A cowhorn dilator was placed in the defect to control the aorta and cardiopulmonary bypass (CPB) was re-initiated.

Now with some control of the aorta, a sternal retractor was placed and the aorta was dissected circumferentially. Under a second duration of hypothermic circulatory arrest. A large, bovine pericardial patch was then sewn in running fashion around the defect. This appeared to achieve hemostasis at the defect site and the patient was rewarmed and weaned off of $\mathrm{CPB}$ without difficulty. The right groin and left anterior thoracotomy were closed in standard fashion.

The decision was made to pack the chest due to mild coagulopathy and prior complex closure. The patient remained intubated, sedated, and on vasopressors for the duration of the postoperative period. Total time on cardiopulmonary bypass was 171 minutes, with 24 minutes (6 minutes on entry, and 18 minutes while sewing patch) of total hypothermic circulatory arrest time. 
His postoperative course was complicated by seizures thought to be due to air embolism on subsequent MRI. His operative wound cultures grew Candida albicans. Although he underwent successful washout and closure of his flaps, he later become profoundly hypotensive with acute hemorrhage from his chest tubes. Upon emergently opening his chest, 3 liters of blood was noted in his mediastinum with bleeding from the left side of the bovine pericardial patch. The aorta quality was poor. Hemostasis was achieved with primary repair. Temporary closure was performed and the chest was packed. The patient was weaned off pressors. Ultimately, given the complex disease and concerning neurological status, the decision was made to transition to comfort care and the patient expired soon after.

\section{Discussion}

Herein, we present a complicated case of an aortic cannulation site pseudoaneurysm that occurred after DSWI following an AVR. The original sternal infection was unlikely treated sufficiently and was most likely the cause of the late development of pseudoaneurysm. Ultimately, he had a challenging diagnosis and anatomy making emergent repair challenging.

The surgical repair of aortic pseudoaneurysms are variable and largely patient dependent ${ }^{4-6}$. In cases with a small defect and no sign of infection, a simple graft, composite replacement, patch, or primary closure with a simple suture can be considered ${ }^{4,7}$. In the presence of active infection, aggressive debridement of the pseudoaneurysm including any grossly infected aorta with closure and repair using allograft, bovine or autologous pericardial patches ${ }^{4}$. Our patient presented with a fungal mediastinal infection. Candida has found to be particularly associated with significantly worse outcomes, with twice as high mortality than bacterial causes of $\mathrm{DSWI}^{8}$. It is likely that Candida mediastinal infection would require more aggressive debridement and replacement of the aorta. In a more elective setting, more complete aortic resection and replacement with homograft or rifampin-soaked graft, may be more resistant to rebleed and/ or reinfection.

When considering approach to re-sternotomy in these patients, femoral artery cannulation, left ventricular venting, hypothermia, and circulatory arrest are typically required ${ }^{3,7,9,10}$. In a non-emergent setting, deeper cooling, the use of $\mathrm{CO} 2$ on the field, and the ability to place an aortic root vent with sufficient exposure of the aorta all may have been beneficial in preventing air embolism ${ }^{11}$.

In summary, vigilance following DSWI should be maintained for several months postoperatively. Recurrent infection should warrant urgent imaging and a thoughtful approach when feasible. Multidisciplinary approaches should be considered when feasible for optimal chances of survival.

\section{Conflicts of Interest/Disclosures:}

Ailawadi is a consultant for Medtronic, Admedus, Gore, Edwards, Abbott, and Atricure (all $<5 \mathrm{~K})$. The other authors have no disclosures.

\section{Author Contributions:}

All authors contributed significantly to the drafting and revision of this manuscript.

\section{References}

1. Katsumata T, Moorjani N, Vaccari G, Westaby S. Mediastinal false aneurysm after thoracic aortic surgery. The Annals of Thoracic Surgery. 2000;70(2):547-552.

2. Fillmore AJ, Valentine RJ. Surgical mortality in patients with infected aortic aneurysms. Journal of the American College of Surgeons. 2003;196(3):435-441.

3. El Oumeiri B, Louagie Y, Buche M. Reoperation for ascending aorta false aneurysm using deep hypothermia and circulatory arrest. Interact CardioVasc Thorac Surg. 2011;12(4):605-608.

4. Atik FA, Navia JL, Svensson LG, et al. Surgical treatment of pseudoaneurysm of the thoracic aorta. The Journal of Thoracic and Cardiovascular Surgery. 2006;132(2):379-385.e1. 
5. Settepani F, Muretti M, Barbone A, et al. Reoperation for Aortic False Aneurysms: Our Experience and Strategy for Safe Resternotomy. Journal of Cardiac Surgery. 2008;23(3):216-220.

6. Parihar B, Choudhary LSD, Madhu AP, Alpha MK, Thankachen R, Shukla V. Pseudoaneurysm of Ascending Aorta After Aortic Valve Replacement. The Annals of Thoracic Surgery. 2005;79(2):705-707.

7. Villavicencio MA, Orszulak TA, Sundt TM, et al. Thoracic Aorta False Aneurysm: What Surgical Strategy Should Be Recommended? The Annals of Thoracic Surgery. 2006;82(1):81-89.

8. Modrau IS, Ejlertsen T, Rasmussen BS. Emerging Role of Candida in Deep Sternal Wound Infection. The Annals of Thoracic Surgery. 2009;88(6):1905-1909.

9. Cohn WE. A Transthoracic, Left Ventricular Vent Facilitates Challenging Sternal Reentry. The Annals of Thoracic Surgery. 2010;90(2):679-680.

10. Dumont E, Carrier M, Cartier R, et al. Repair of aortic false aneurysm using deep hypothermia and circulatory arrest. The Annals of Thoracic Surgery. 2004;78(1):117-120.

11. Orihashi K, Ueda T. "De-airing" in open heart surgery: report from the CVSAP nation-wide survey and literature review. Gen Thorac Cardiovasc Surg. 2019;67(10):823-834.
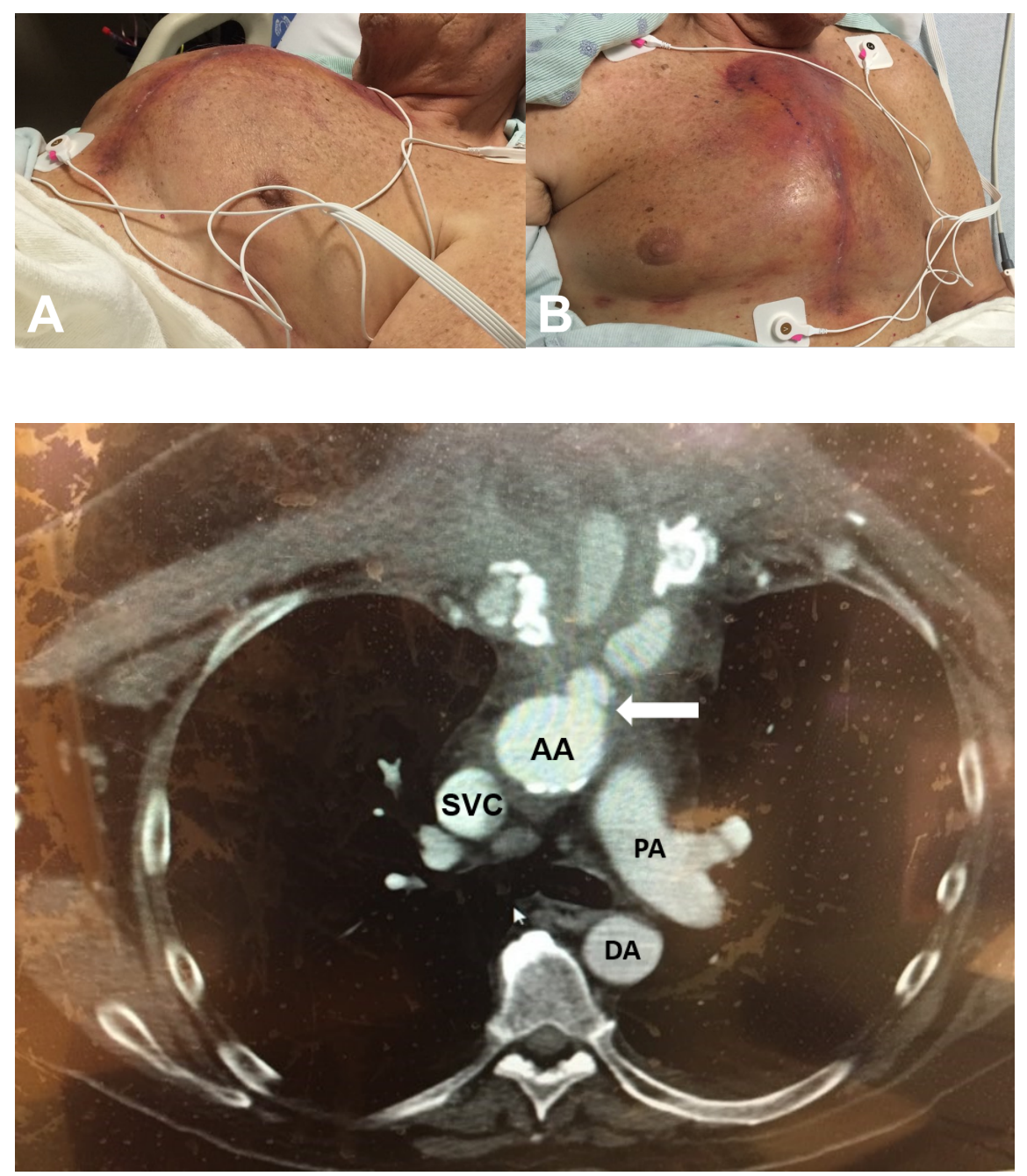


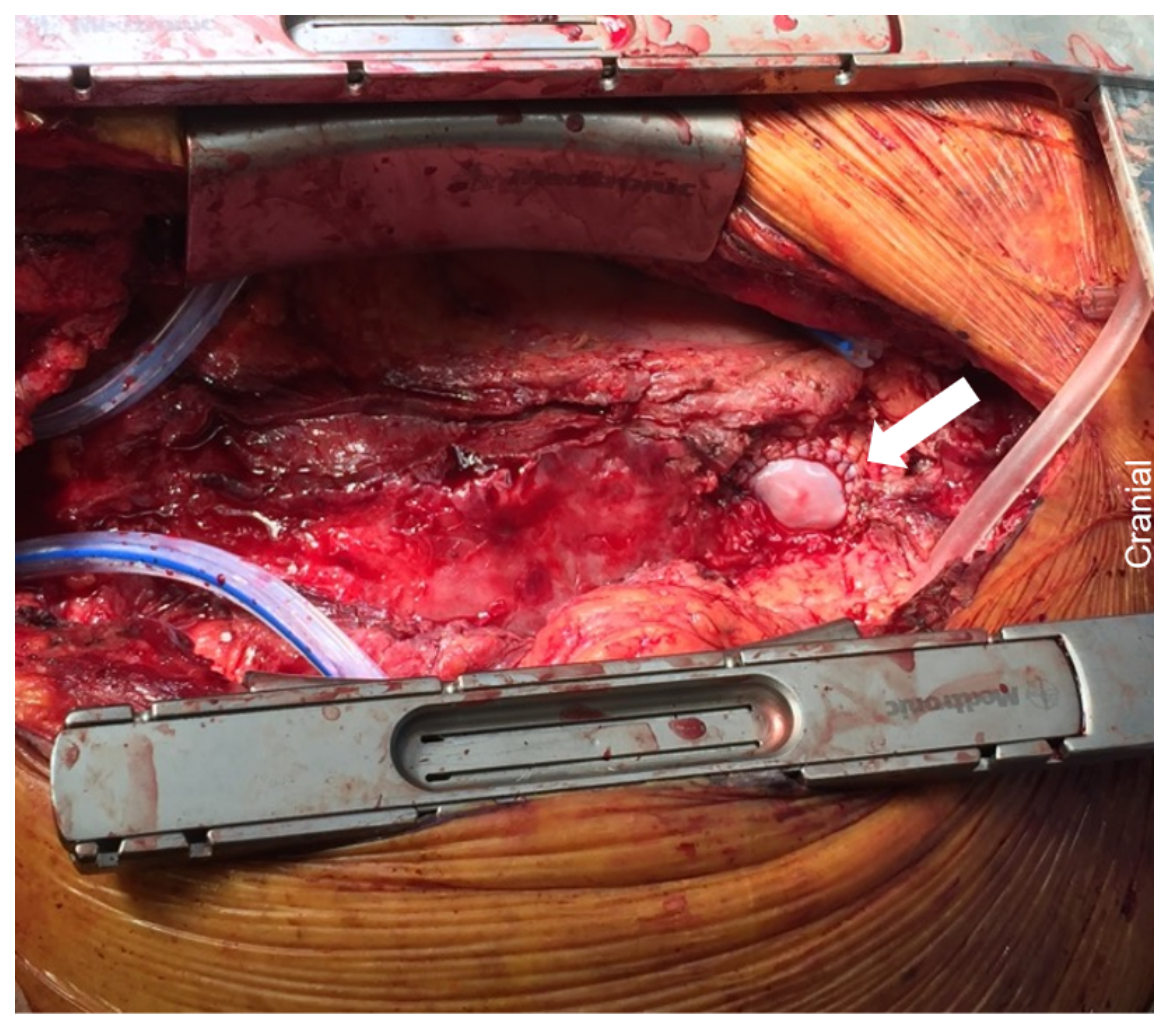

